\title{
Effect of Sensor Mobility and Channel Fading on Wireless Sensor Network Clustering Algorithms
}

\author{
Ahmed H. Gabr \\ Department of Computer Engineering \\ Arab Academy for Science \& technology \\ Cairo, Egypt \\ E-mail: ahgabr@hotmail.com
}

\author{
Sherif Keshk \\ Department of Electronics and Electrical Communication \\ Mansoura University \\ Cairo, Egypt \\ E-mail: shkishk@gmail.com
}

\author{
Aliaa A. A. Youssif \\ Department of Computer Engineering \\ Arab Academy for Science \& technology \\ Cairo, Egypt \\ E-mail: aliaay@aast.edu.eg
}

Received: November 14, 2011

Accepted: December 8, 2011

Published: January 1, 2012

doi: $10.5539 /$ cis.v5n $1 \mathrm{p} 20$

URL: http://dx.doi.org/10.5539/cis.v5n1p20

\begin{abstract}
Clustering is an effective topology approach in wireless sensor network, which can increase network lifetime and scalability. Either Node Mobility or Channel fading has a negative impact on various clustering protocol. In case of Node Mobility when all sensor nodes are mobile the current nearest cluster head may be the farthest one for the sensor node when message transmission phase starts. In the present research the received signal strength is used to estimate the sensor location. Consequently, channel fading affects the path loss between the nodes thus affecting the estimated distance between them. This paper introduces a new clustering protocol which is built on Adaptive Decentralized re-clustering protocol called E-ADRP (Enhanced Adaptive Decentralized re-clustering protocol). Simulations are performed to test the effect of node mobility using Random Walk Mobility model (RWM) on Low Energy Adaptive Clustering Hierarchy (LEACH) and Enhanced Adaptive Decentralized re-clustering protocol (E-ADRP). The simulation results show that the applied mobility on LEACH affected the network lifetime and energy dissipation negatively while in contrast E-ADRP simulation results were much better. On the other side, Rayleigh channel model also was applied on LEACH and E-ADRP clustering algorithms. The simulation results show that network lifetime and energy dissipation at mobile nodes were nearly stable compared to static nodes in case of E-ADRP while in case of LEACH mobile nodes were negatively affected by rate up to $24 \%$ less than static nodes, at fading E-ADRP and LEACH were both negatively affected where E-ADRP was affected by rate up to $40 \%$ less than static nodes and LEACH was affected by rate up to $50 \%$ less than static nodes.
\end{abstract}

Keywords: Wireless Sensor Network, Clustering algorithm, Mobility, Fading

\section{Introduction}

Wireless sensor networks (WSNs) have gained world-wide attention in recent years. These networks are composed of tiny and compact devices called sensor nodes which contain three components: a sensing subsystem for data acquisition from the physical surrounding environment, a processing subsystem for local data 
processing and storage, and a wireless communication subsystem for data transmission (Al-Karaki \& Kamal, 2004). These Sensor Nodes are deployed in a remote area to detect certain phenomena then collect the information of interest, perform local processing for these data and forward that data to base station (BS) directly or through a neighboring relay node. Direct interaction with physical phenomena resulted in the development of many applications for wireless sensor networks such as healthcare, target tracking, infrastructure monitoring, military applications (Yick, Mukherjee, \& Ghosal, 2008). WSNs are able to organize themselves and work unattended so sensors must rely only on their battery for communication and information gathering. Wireless sensor nodes have many limitations, including simple processing power, limited power source and little storage. Power conservation has been well recognized by the sensor network community as a critical factor to prolong the network lifetime. Wireless channel path loss is the major source of energy loss in WSN. Therefore, energy efficient topology architectures and communication protocols are highly desirable. Clustering has proved to be an efficient topology for saving energy in recent years. Nodes are organized into separated groups not overlapped called clusters. Each cluster has a coordinator referred to as cluster head $(\mathrm{CH})$ and a number of member nodes, member nodes transfer data of sensed information to $\mathrm{CH}$ according to time division multiplexing (TDMA) to save energy, then $\mathrm{CH}$ aggregate data messages and send it to BS (Akkaya \& Younis, 2005; Abbasi \& Younis, 2007; Muruganathan, Ma, Bhasin, \& Fapojuwo, 2005). In recent years several algorithms have been proposed for clustering WSNs. Low-energy Adaptive Cluster Hierarchy (LEACH) was proposed by W. Heinzelman et al. (Heinzelman, Chandrakasan, \& Balakrishnan, 2002) as a single-hop self-organized clustering algorithm for wireless sensor networks. In LEACH every sensor node determine if it will be $\mathrm{CH}$ for current round or not according to probability threshold and advertise a message to all sensor nodes that it become the $\mathrm{CH}$ of current round. The remaining sensor nodes receive the broadcast from one or more CHs and make their association decision based on minimum communication cost. The main disadvantage of LEACH is that it has a predefined number of clusters and it does not guarantee good $\mathrm{CH}$ distribution. The Hybrid Energy Efficient Distributed Protocol (HEED) (Younis \& Fahmy, 2004) provides balanced cluster heads and smaller sized clusters. In HEED the $\mathrm{CH}$ election process is performed using a hybrid metric consisting of residual energy and communication cost. The primary parameter is residual energy and the secondary parameter is the average minimum reach ability power (AMRP). Threshold-sensitive Energy Efficient sensor Network protocol (TEEN) (Manjeshwar \& Agarwal, 2001) is designed for time-critical applications. In TEEN the $\mathrm{CH}$ broadcasts two thresholds to the nodes, hard and soft thresholds for sensed attributes. The hard threshold aims to reduce the number of transmissions by allowing the nodes to transmit only when the sensed attribute is in the range of interest. The soft threshold reduces the number of transmissions if there is little or no change in the value of sensed attribute. Adaptive Decentralized re-clustering protocol (ADRP) (Bajaber \& Awan, 2011) provides an efficient way to improve the scalability and lifetime of Wireless Sensor Network. In ADRP cluster head and next head are elected based on residual energy of each node and average energy of each cluster. In case of mobility random walk mobility model (RWM) (Camp, Boleng, \& Davies, 2002) is used, where each sensor node randomly choose a speed within a given range and also a direction, then after moving a constant time or distance, each sensor node calculates a new speed and direction to modify its current position. For every new interval t, each node randomly and uniformly chooses its new direction $\theta(\mathrm{t})$ from $[0,2 \pi]$. In similar way, the new speed follows a uniform distribution or a Gaussian distribution from $\left[0, V_{\max }\right]$. In case the node moves according to the above rules and reaches the boundary of simulation field, the leaving node is bounced back to the simulation field with the angle of $\theta(\mathrm{t})$ or $\pi-\theta(\mathrm{t})$, respectively. Rayleigh fading model is used which affects the received signal strength between any two nodes thus affects the distance calculated between them. The paper is organized as follows: section 2 defines system model, section 3 provides an overview of the used clustering techniques, section 4 presents the simulation setup and results and section 5 concludes the paper.

\section{System Model of Clustering Algorithm}

The Wireless Sensor Network consists of N sensor nodes which are deployed randomly in the sensing field. As shown in Figure 1, each node is assigned as a cluster member and every cluster has an elected cluster head which collect sensor data from other nodes in the clusters and transfer the aggregated data to the base station.

The following assumptions are considered in the proposed model:

(1) There is a base station located far away from the sensing field.

(2) Each sensor node is assigned a unique identifier (ID).

(3) Each sensor node has power control and the ability to transmit data to any other sensor node or directly to the base station. 
(4) All the sensor nodes are location aware. To get the information, sensor nodes can use GPS or other location detect scheme.

It is assumed that the model may be subjected to fading where Rayleigh channel model is used to analyze that probability in the simulation. Rayleigh distribution (Garg, 2007) is commonly used to describe the statistical time varying nature of the received envelope of a flat fading signal. It is well known that the envelope of the sum of two quadrature Gaussian noise signals obeys a Rayleigh distribution.

$$
z(\mathrm{t})=|\mathrm{r}(\mathrm{t})|=\sqrt{r_{I}^{2}(t)+r_{Q}^{2}(t)}
$$

$z(\mathrm{t})$ is signal envelope, $\mathrm{r}(\mathrm{t})$ is the received signal, $r_{I}$ and $r_{Q}$ are both zero-mean Gaussian random variables.

If it is assumed a variance of $\sigma^{2}$ for both in-phase and quadrature components then the signal envelope is Rayleigh distributed with distribution

$$
\mathrm{P} z(\mathrm{r})=\frac{2 r}{P_{r}} \exp \left(-\frac{r^{2}}{P_{r}}\right)=\frac{r}{\sigma^{2}} \exp \left(-\frac{r^{2}}{2 \sigma^{2}}\right), x \geq 0
$$

Where $\mathrm{P}_{\mathrm{r}}=\sum_{n} E\left[\alpha_{n}^{2}\right]=2 \sigma^{2}$ is the average received signal power of the signal, i.e. the received power based on path loss and shadowing alone.

Power distribution is obtained by making the change of variables $Z^{2}(\mathrm{t})=|\mathrm{r}(\mathrm{t})|^{2}$ where it obtains the formula below

$$
\mathrm{Pz}^{2}(\mathrm{x})=\frac{1}{P_{r}} e^{-\frac{x}{P_{r}}}=\frac{1}{2 \sigma^{2}} e^{-\frac{x}{\sigma^{2}}}, x \geq 0
$$

Thus, the received signal power is exponentially distributed with mean $2 \sigma^{2}$.

\section{Clustering Techniques}

In recent years clustering for ad hoc and WSNs have been a popular area of research and several algorithms have been proposed. These techniques can be classified in different ways such as clustering method (distributed, centralized), network architecture (single-hop, multi-hop), clustering objective (energy efficiency, coverage) or $\mathrm{CH}$ selection method (random, deterministic). Here it is focused on two single-hop clustering algorithms, Low Energy Adaptive Clustering Hierarchy (LEACH) and Enhanced Adaptive Decentralized re-clustering protocol (E-ADRP). LEACH is an effective distributed clustering algorithm which has been previously used by many researchers where cluster head is elected based on probability threshold. E-ADRP uses a centralized clustering architecture which makes use of location and residual energy to elect its cluster head. Both techniques will be farther discussed in more details in the below sections.

\subsection{Low Energy Adaptive Clustering Hierarchy (LEACH) (Heinzelman, Chandrakasan, \& Balakrishnan, 2000)}

Leach Protocol is the first hierarchical cluster-based routing protocol for wireless sensor network which partitions the nodes into clusters, in each cluster a dedicated node called Cluster Head $(\mathrm{CH})$ is aggregating the data sent by cluster members. In setup phase, $\mathrm{LEACH}$ forms clusters by using a distributed algorithm, where nodes make autonomous decisions without any centralized control. Each node decides independent of other nodes if it will become a $\mathrm{CH}$ or not. This decision is based on the threshold $\mathrm{T}(\mathrm{n})$ given by,

$$
\mathrm{T}(\mathrm{n})=\left\{\begin{array}{c|l}
\frac{P}{1-P\left(\operatorname{rmod} \frac{1}{P}\right)} & \text { If } \mathrm{n} \in \mathrm{G} \\
0 & \text { Otherwise }
\end{array}\right.
$$

Where $p$ is the predetermined percentage of cluster heads (e.g., $p=0.05$ ), $r$ is the current round, and $G$ is the set of nodes that have not been cluster heads in the last $1 / p$ rounds.

Once the nodes have elected themselves to be cluster heads using the probabilities cluster head broadcasts an advertisement message to the rest of the nodes using a nonpersistent carrier-sense multiple access (CSMA) MAC protocol. This message is a small message containing the node's ID and a header that distinguishes this message as an announcement message. Non-CH nodes pick the advertisement packet with the strongest received signal strength and inform the $\mathrm{CH}$ that they become a member to that cluster with "join packet" contains their IDs using nonpersistent CSMA MAC protocol. This message is again a short message, consisting of the node's ID and the cluster head's ID. Based on all messages received within the cluster, the $\mathrm{CH}$ creates a TDMA schedule and broadcast the TDMA table to cluster members. After the TDMA schedule is known by all nodes in the cluster, 
the set-up phase is complete and the steady-state begins. At steady state $\mathrm{CH}$ receives data sent by cluster members during their allocated transmission slot then Cluster Head aggregates the data messages received, eliminate redundancy to reduce the amount of data received then at last send the aggregated data to base station.

\subsection{Enhanced Adaptive Decentralized Re-clustering Protocol (E-ADRP)}

E-ADRP is a clustering protocol for wireless sensor networks where network activity is organized into rounds, where each round has two phases: initial phase and cycle phase. At first all sensor nodes in the network send their current position and remaining energy level to base station then the base station use them to split the network into clusters and determine the cluster head by minimizing the total sum of the distances between the cluster heads and sensor nodes. After cluster is formed base station computes the average energy for each cluster in the network to determine one next head, and sensor nodes that have energy storage below this average cannot become a next head for this round. Once the cluster head and next head is determined base station broadcast this information to all sensor nodes. Then TDMA schedule is created by cluster head which gives every sensor a specified time slot to send its data, after sensors send the data cluster head aggregate these data packets and eliminate redundancy before sending it to base station. At the end of this phase each node selects next head and switch to as new cluster head. Re-clustering occurs periodically.

\section{Simulation and Discussion}

In the provided simulation, the effect of random sensor nodes mobility and channel fading is discussed for both $\mathrm{LEACH}$ and E-ADRP clustering protocols. C language is used to simulate the system in terms of the following metrics:

- Network lifetime: this metric shows the number of sensor nodes that die over the time of operation. base station.

- Received data messages: this metric shows the number of data messages successfully delivered to the

- Energy dissipation: this metric shows the energy consumption over activity time.

The simulations were configured on network size with 100 nodes randomly distributed. The same radio parameters as Heinzelman et al. (Al-Karaki \& Kamal, 2004; Heinzelman, Chandrakasan, \& Balakrishnan, 2002) is used to compute energy consumption for each sending and receiving. The energy used to send $n$-bit data for a distance $d$ for each sensor node is

$$
\mathrm{E}_{\mathrm{Tx}}(\mathrm{n}, \mathrm{d})=\mathrm{nE}_{\text {elec }}+\mathrm{n} \varepsilon f s \mathrm{~d}^{2}
$$

Where $\mathrm{E}_{\mathrm{Tx}}$ is energy dissipated for transmission, $\mathrm{n}$ is the size of packet, $\mathrm{E}_{\text {elec }}$ is the energy dissipated per bit to run the transmitter or receiver circuit, $\varepsilon f s$ is power loss for free space and $\mathrm{d}$ is the distance between nodes.

The energy used to receive $n$-bit data a distance $d$ for each sensor node is

$$
E_{R x}(n)=n E_{\text {elec }}
$$

Where $E_{R x}$ is energy dissipated for reception, $\mathrm{n}$ is the size of packet, $E_{\text {elec }}$ is the energy dissipated per bit to run the transmitter or receiver circuit.

The energy for data aggregation is

$$
\mathrm{E}_{\mathrm{DA}}=5 \mathrm{~nJ} / \mathrm{bit} / \mathrm{signal}
$$

Table 1 summarizes parameters used in the simulation.

The simulation is performed to analyze the network life time which is defined by using metrics like FD (First Node Dies), HD (Half of the Nodes Alive), and LD (Last Node Dies) (Handy, Haase, \& Timmermann, 2002). Since more than one node is necessary to perform the clustering algorithm. The LD represents overall lifetime of wireless sensor network when $80 \%$ of sensor nodes die.

Different Network Size with different number of clusters are simulated for both LEACH and E-ADRP to analyze the network in case of static nodes, mobile nodes and static nodes affected by channel fading which will be represented in section 4.1, also a sample will be discussed in details in section 4.2. Mobile nodes are simulated at speeds $2 \mathrm{~m} / \mathrm{s}$ and $5 \mathrm{~m} / \mathrm{s}$ where speed $2 \mathrm{~m} / \mathrm{s}$ simulates normal walk of a patient inside a hospital were sensor acts as a tracking device, also mobile nodes are simulated under speed $5 \mathrm{~m} / \mathrm{s}$ which tracks the movement of small animals like squirrel in a forest. At fading two mean variables (mu) 4000 and 5000 are assumed which are used as a mean for the exponential random numbers which produces noise to the received signal.

\subsection{Simulating Different Network Size}

Table 2 shows the performance of E-ADRP and LEACH at different network size where at first part when 
number of clusters at E-ADRP equal 20 and for LEACH equal 5 and network size was $(X=100 \mathrm{~m}, \mathrm{Y}=100 \mathrm{~m})$ LEACH performance was better than E-ADRP at static nodes where E-ADRP first node died at round 344 while LEACH died at round 590, that is because LEACH uses a predefined number of clusters equal to 5 which is the optimum number of clusters as per Heinzelman et al. (Heinzelman, Chandrakasan, \& Balakrishnan, 2002). In case of E-ADRP number of clusters is predefined by 20 which dissipate a large amount of energy. On other side when the network size is enlarged to size $(X=200 \mathrm{~m}, \mathrm{Y}=200 \mathrm{~m})$ the gap between the two protocols gets more close where at E-ADRP first node died at round 198 and for LEACH it died at round 203 that is because when region size increases the distance between the sensors and its cluster head also increase so cluster members loses a bigger amount of energy to reach its cluster head. At region size $(X=50 \mathrm{~m}, \mathrm{Y}=50 \mathrm{~m}) \mathrm{LEACH}$ was better than E-ADRP where sensors deployed on region become closer in distance to each other. The performance of sensors are affected by sensor mobility but as it is seen LEACH is more affected by mobility than E-ADRP, that is because LEACH randomly choose its cluster heads according to probability threshold which may determine cluster heads to be at the edge of its own clusters so mobility may lead to probably movement of cluster head far away maybe double the distance away from its cluster members while in E-ADRP re-clustering occurs which choose the best cluster head for all clusters based on best residual energy and position. At fading both protocols are affected since fading affects the path loss between any two nodes thus affecting the estimated distance between them, also it can be seen that LEACH is more negatively affected than E-ADRP by comparing to first node dead in case of static nodes.

At second part when number of clusters at both E-ADRP and LEACH are equal to 20 E-ADRP performance was better than LEACH at network size $(\mathrm{X}=100 \mathrm{~m}, \mathrm{Y}=100 \mathrm{~m})$ where at static nodes E-ADRP first node died at round 344 while LEACH died at round 327 and at region $(X=200 \mathrm{~m}, \mathrm{Y}=200 \mathrm{~m})$ E-ADRP first node died at round 198 while LEACH died at round 141. That is because of previous mentioned characteristics of both protocols. Mobility affects both protocols but LEACH was much more affected at higher speed for both regions, fading have a very bad effect on both protocols but LEACH was much more negatively affected.

At third part when number of clusters at both E-ADRP and LEACH were equal to 5 . As it is seen at network size $(\mathrm{X}=100 \mathrm{~m}, \mathrm{Y}=100 \mathrm{~m}) \mathrm{LEACH}$ performance is better than E-ADRP where LEACH optimum clusters is 5 as per Heinzelman et al. (Heinzelman, Chandrakasan, \& Balakrishnan, 2002). When region is enlarged to network size $(\mathrm{X}=200 \mathrm{~m}, \mathrm{Y}=200 \mathrm{~m})$ the gap between the two protocols become closer as it is shown below, that is because enlarging the network size makes the distances between sensors to be farther than it was at network size $(\mathrm{X}=100 \mathrm{~m}, \mathrm{Y}=100 \mathrm{~m})$ which negatively affects the performance of LEACH while E-ADRP still be better.

At fourth part when number of clusters at both E-ADRP and LEACH are equal to 10 LEACH was negatively affected by increasing number of clusters at region size $(X=100 \mathrm{~m}, \mathrm{Y}=100 \mathrm{~m})$ than it was at number of clusters equal 5 so first node dies at round 521 instead of 589 in case of static node at number of clusters equal 5 and E-ADRP was nearly stable where first between both sizes. When network size is increased to $(X=200 \mathrm{~m}$, $\mathrm{Y}=200 \mathrm{~m}$ ) both protocols where affected but LEACH was more affected than E-ADRP as shown below. Mobility affects both protocols but LEACH was more affected since E-ADRP uses clustering technique in every round where it respites the network into clusters which help in improving its performance while for LEACH clusters are chosen randomly which doesn't help it when mobility occurs. At Fading both protocols where affected but LEACH was much more affected.

Table 2 illustrates first node dead round for LEACH and E-ADRP at different network size.

\subsection{Sample of Performed Simulation}

\subsubsection{Effect of Sensor Mobility}

In the first set of experiments we test the effect of mobility on the performance of LEACH and E-ADRP at speed $2 \mathrm{~m} / \mathrm{s}$. Putting into consideration that the number of clusters for LEACH is predefined with a probability factor which is fixed. 5 clusters are used for LEACH which is the optimum number of cluster as per Heinzelman et al. (Heinzelman, Chandrakasan, \& Balakrishnan, 2002), while for E-ADRP K-means clustering is used with number of clusters equal 20 . These clusters are decreased by one and re-clustering occurs whenever there is no sensor in the cluster or no cluster head able to send aggregated data to base station due to its low energy. Figure 2(a-b) shows the number of dead nodes versus the number of rounds. The Figures show the effect of mobility on E-ADRP and LEACH where the dashed curve represents the number of dead nodes in case of static sensors and the solid line curve represents number of dead nodes after mobility occurs. Mobility of sensors occurs after determining the cluster heads in the initial phase. As it is seen in the figure, LEACH was negatively affected due to mobility where it died at round 584 while E-ADRP died at round 375, that is because LEACH determines random cluster heads according to probability threshold which may determine cluster heads to be at the edge of 
its own clusters so mobility may lead to probably movement of cluster head far away maybe double the distance away from its cluster members while in E-ADRP it was better because it performs re-clustering every round to re-split the network into clusters after mobility and then choose the best cluster heads for all clusters based on best residual energy and position of all nodes in the same cluster. Figure 2(c) shows a relation between E-ADRP and LEACH for calculating number of received data packets by base station. As the figure shows E-ADRP sends more data messages than LEACH, which is because in E-ADRP there are more clusters therefore more cluster heads than LEACH. Figure 2(d) shows the relation between the energy dissipation and the number of rounds for both LEACH and E-ADRP. As shown E-ADRP energy is more dissipated than LEACH, the reason for that is that there is more clusters in E-ADRP than LEACH so therefore consumes more power.

In the second set of experiments the effect of increasing sensor speed is tested on the performance of LEACH and E-ADRP at speed $5 \mathrm{~m} / \mathrm{s}$. Figure $3(\mathrm{a}-\mathrm{b})$ shows that the LEACH lifetime is getting shorter while E-ADRP is much more better where first node dies in E-ADRP at round 378 and in LEACH at round 535 which differs from speed $2 \mathrm{~m} / \mathrm{s}$ where it was 375 for E-ADRP and 584 for $\mathrm{LEACH}$, the reason for that is because the distance between cluster head and cluster members is affected due to movement of cluster heads far away from their cluster, also it is seen that E-ADRP has a straight curve at the last part of it while LEACH is more bended, the reason is that E-ADRP distribute the energy between the sensor nodes by positioning cluster heads into the center of clusters and also cluster heads are well chosen to be the most one which have powerful residual energy than all other in the same clusters so at the end of the simulation, sensors would have no great variations in energy so when they die, they die altogether in contrast there is no interference in the position of cluster head in case of LEACH also all cluster heads are chosen randomly according to probability. Figure 3(c) shows that E-ADRP sends more data messages to base station than LEACH but it is more affected by mobility than at a speed of $2 \mathrm{~m} / \mathrm{s}$ where at speed $2 \mathrm{~m} / \mathrm{s}$ E-ADRP sends 15988 packet while in LEACH it sends 4000 packet, at speed $5 \mathrm{~m} / \mathrm{s}$ E-ADRP sends 16000 packet while in LEACH it sends 3978 packet. Figure 3(d) shows the energy dissipated for both E-ADRP and LEACH where the increase of speed affected E-ADRP more than LEACH since E-ADRP sends its location and residual energy every round to base station to perform reclustering which fixes the effect of mobility on sensors.

\subsubsection{Effect of Channel Fading on Clustering}

In the next experiment the effect of channel Rayleigh fading is tested on the performance of LEACH and E-ADRP using fading parameter $(\mathrm{mu}=4000)$. Figure $4(\mathrm{a}-\mathrm{b})$ shows the effect of fading on the system using E-ADRP and LEACH. As the figures show fading affected both E-ADRP and LEACH but LEACH was much more affected where first node died time for E-ADRP at round 233 and for LEACH at round 405, that is because LEACH randomly choose cluster head according to probability threshold, so cluster heads may be at the edge of the cluster not in the center of it to minimize the energy dissipation so it is much more affected by fading than E-ADRP which has a well distributed clusters and all cluster heads are located near center of their clusters. Also, at LEACH cluster heads may be chosen near a certain boundary inside the network region, where cluster members located at the other boundary loses so much power to communicate with its cluster heads, so when fading occurs it may be a problem for cluster members to communicate with their cluster heads or even if they could communicate they would lose a very big amount of energy to reach their cluster heads. Figure 4(c) shows that E-ADRP sends more packets to base station than LEACH, which is because as it is mentioned before the predefined number of clusters at the initial phase differs from each other which affected the sent messages. Figure 4(d) shows the energy dissipated for E-ADRP was more affected than LEACH.

In the last set of experiments the performance of LEACH and E-ADRP using fading parameter $(m u=5000)$ is tested. Figure 5(a-b) shows that both E-ADRP and LEACH where negatively affected where first node died for E-ADRP at round 219 instead of 233 in case 1 and LEACH was at round 373 instead of 405 in case 1.That is of the increase of fading parameter. Figure 5(c) shows that data messages sent by E-ADRP or LEACH to base station are both negatively affected by fading relative to case 1 . Figure 5(d) shows that energy dissipated for E-ADRP and LEACH were both negatively affected relative to case 1.

\section{Conclusion}

In this paper, the effect of sensor mobility and fading is studied on both Enhanced Adaptive Decentralized re-clustering protocol (E-ADRP) and Low Energy Adaptive Clustering Hierarchy (LEACH). Different region dimensions are used with different number of clusters were the simulations clarify that LEACH performance is better than E-ADRP when region dimension is $(X=100, Y=100)$ or less with number of clusters between 5 and 12 while when the region is bigger than $(X=100, Y=100)$ E-ADRP's performance gets better. On other side In case of mobility, simulation showed that at LEACH the system was negatively affected, the reason for that is that 
LEACH randomly chooses cluster heads where the nearest cluster members joins this cluster. Organizing clusters in the mentioned way leads to different problems. Firstly, cluster heads may be located at the edge of cluster where mobility move the cluster head far away of cluster which may double the distance between the cluster head and its members which wastes a lot of energy from cluster members to reach it. Secondly, clusters have different variations in size which leads to an instability in the system where clusters may die faster than others. In contrast E-ADRP base station well split the network according to location and residual energy of sensors nodes, also cluster head are chosen to be the most powerful energy and the most nearest one to the center of the cluster, this helps to reduce energy consumption after mobility occurs also E-ADRP perform re-clustering every round to restructure itself due to mobility. In case of fading, simulation showed that both E-ADRP and LEACH were both affected but LEACH was more affected due to reasons mentioned before, another experiment is performed to increase the variance of fading where both E-ADRP and LEACH were negatively affected due to that increase.

\section{References}

Abbasi, A.A, \& Younis, M. (2007). A survey on clustering algorithms for wireless sensor networks. Computer Communications, 30, 2826-2841. http://dx.doi.org/10.1016/j.comcom.2007.05.024

Akkaya, K., \& Younis, M. (2005). A survey on routing protocols for wireless sensor networks. Journal of Ad Hoc Networks, 3(3), 325-349. http://dx.doi.org/10.1016/j.adhoc.2003.09.010

Al-Karaki, J., \& Kamal, A. (2004). Routing techniques in wireless sensor networks: a survey. Wireless Communications, 11(6), 6-28. http://dx.doi.org/10.1109/MWC.2004.1368893

Bajaber, F., \& Awan, I. (2011). Adaptive decentralized re-clustering protocol for wireless sensorNetworks. Journal of computer and system science, 77(2), 282-292. http://dx.doi.org/10.1016/j.jcss.2010.01.007

Camp, T., Boleng, J., \& Davies, V. (2002). A Survey of Mobility Models for Ad Hoc Network Research. Wireless Communication and Mobile Computing (WCMC): Special issue on Mobile Ad Hoc Networking: Research, Trends and Applications, 2, 483-502. http://dx.doi.org/10.1002/wcm.72

Garg, V. (2007). Wireless communications and networking. San Francisco: Morgan-Kaufmann, (Chapter 3).

Handy, M.J., Haase, M., \& Timmermann, D. (2002). Low energy adaptive clustering hierarchy with deterministic cluster-head selection. Proceedings 4th International Workshop on Mobile and Wireless Communications Network, 368-372. http://dx.doi.org/10.1109/MWCN.2002.1045790

Heinzelman, W., Chandrakasan, A., \& Balakrishnan, H. (2002). An application specific protocol architecture for wireless micro sensor networks. IEEE Transactions on Wireless Communications, 1(4), 660-670. http://dx.doi.org/10.1109/TWC.2002.804190

Heinzelman, W.R., Chandrakasan, A., \& Balakrishnan, H. (2000). Energy efficient communication protocol for wireless microsensor networks. Proceedings of the $33^{\text {rd }}$ Hawaii International Conference on System Science, 2. http://dx.doi.org/10.1109/HICSS.2000.926982

Manjeshwar, A., \& Agarwal, D.P. (2001). TEEN: a routing protocol for enhanced efficiency in wireless sensor networks.15th International Parallel and Distributed Processing Symposium (IPDPS'01), 3, 2009-2015. http://dx.doi.org/10.1109/IPDPS.2001.925197

Muruganathan, S.D., Ma, D.C.F., Bhasin, R.I., \& Fapojuwo, A.O. (2005). A centralized energy-efficient routing protocol for wireless sensor networks. IEEE Communications Magazine, 43(3), S8-13. http://dx.doi.org/10.1109/MCOM.2005.1404592

Yick, J., Mukherjee, B., \& Ghosal, D. (2008). Wireless sensor network survey. Computer Networks, 52(12), 2292-2330. http://dx.doi.org/10.1016/j.comnet.2008.04.002

Younis, O., \& Fahmy, S. (2004). Heed: a hybrid, energy-efficient, distributed clustering approach for ad hoc sensor networks. IEEE Transactions on Mobile Computing, 23(4), 366-379. http://dx.doi.org/10.1109/TMC.2004.41 
Table 1. Simulation parameter

\begin{tabular}{|l|l|}
\hline Parameter & Value \\
\hline Number of nodes & 100 \\
\hline $\mathrm{E}_{\mathrm{elec}}$ & $50 \mathrm{n} / \mathrm{bit}$ \\
\hline$\varepsilon_{\mathrm{fs}}$ & $10 \mathrm{p} / \mathrm{bit} / \mathrm{m}^{2}$ \\
\hline Initial Energy & 1 joule \\
\hline Data packet size & 500 bytes \\
\hline Info packet size & 25 bytes \\
\hline
\end{tabular}

Table 2. First node dead round for LEACH and E-ADRP at different network size

\begin{tabular}{|c|c|c|c|c|c|}
\hline \multicolumn{6}{|c|}{ First node dead roumd tor both probcols at num ber of chisters equal 5 tor LEACH amd 20 for E-ADRP } \\
\hline Network Size & Static & Velocity $2 \mathrm{~m} / \mathrm{s}$ & Velocity $5 \mathrm{~m} / \mathrm{s}$ & Rayleigh $(4000)$ & Rayleigh $(5000)$ \\
\hline$X=100 \quad Y=100$ & $\begin{array}{l}\text { ADRP } 344 \\
\text { LEACH } 590\end{array}$ & $\begin{array}{ll}\text { ADRP } & 375 \\
\text { LEACH } & 584\end{array}$ & $\begin{array}{l}\text { ADRP } 378 \\
\text { LEACH } 535\end{array}$ & $\begin{array}{l}\text { ADRP } 233 \\
\text { LEACH } 405\end{array}$ & $\begin{array}{l}\text { P } 219 \\
\text { CH } 373\end{array}$ \\
\hline & Static & $\operatorname{ty} 2 \mathrm{~m} / \mathrm{s}$ & Velocity $5 \mathrm{~m} / \mathrm{s}$ & Rayleigh $(4000)$ & $\operatorname{gh}(5000)$ \\
\hline & ADRP 198 & ADRP 200 & ADRP 195 & ADRP 118 & P 128 \\
\hline$X=200 \quad Y=200$ & LEACH 203 & LEACH 204 & LEACH 17 & LEACH 1 & $r H$ \\
\hline & Stati & $2 \mathrm{~m} / \mathrm{s}$ & $\begin{array}{l}\text { Vel } \\
A D\end{array}$ & 0) & $700)$ \\
\hline$X=50 \quad Y=50$ & LEACH 1361 & LEACH 1357 & LEACH 1305 & LEACH 1361 & LEACH 1361 \\
\hline \multicolumn{6}{|c|}{ First node de ad round for both protocok at number of clusters equal 20} \\
\hline Network Size & 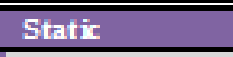 & 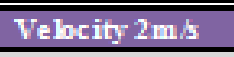 & $\overline{\mathrm{Ve}}$ & 00) & 000) \\
\hline $\mathrm{X}=100 \quad \mathrm{Y}=100$ & $\begin{array}{l}\text { ADRP } 344 \\
\text { LEACH } 327\end{array}$ & $\begin{array}{ll}\text { ADRP } & 375 \\
\text { LEACH } 333\end{array}$ & $\begin{array}{l}\text { ADRP } 378 \\
\text { LEACH } 337\end{array}$ & $\begin{array}{l}233 \\
\mathrm{H} 150\end{array}$ & $\begin{array}{l}219 \\
\mathrm{H} \quad 157\end{array}$ \\
\hline & Sta & $2 \mathrm{~m} / \mathrm{s}$ & $5 \mathrm{~m} / \mathrm{s}$ & $(4000)$ & $(5000)$ \\
\hline & ADRP 198 & ADRP 204 & ADRP 195 & ADRP 118 & ADRP 128 \\
\hline $\mathrm{X}=200 \mathrm{Y}=200$ & LEACH 141 & LEACH 125 & LEACH 113 & LEACH 62 & LEACH 59 \\
\hline \multicolumn{6}{|c|}{ cots at number of clus ters equal } \\
\hline Network Sive & Static & city $2 \mathrm{~ms}$ & $\bar{v}$ & $\operatorname{eigh}(4000)$ & vleigh $(5000)$ \\
\hline$X=100 \quad Y=100$ & $\begin{array}{l}\text { ADRP } 338 \\
\text { LEACH } 589 \\
\text { Static }\end{array}$ & $\begin{array}{l}\text { ADRP } 347 \\
\text { LEACH } 584\end{array}$ & $\begin{array}{l}\text { ADRP } 339 \\
\text { LEACH } 535 \\
\text { Velocity } 5 \mathrm{~m} / \mathrm{s}\end{array}$ & $\begin{array}{l}\text { ADRP } 228 \\
\text { LEACH } 405\end{array}$ & $\begin{array}{l}\text { ADRP } 223 \\
\text { LEACH } 399 \\
\text { Ravleigh } 50000\end{array}$ \\
\hline & & 198 & 196 & 120 & 118 \\
\hline$X=200 \quad Y=200$ & & LEACH 200 & LEACH 173 & LEACH 103 & 99 \\
\hline \multicolumn{6}{|c|}{ und for both protocok at number of clusters equal 10} \\
\hline & & 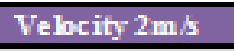 & & & $h(5000)$ \\
\hline \multirow{4}{*}{$X=100 \quad Y=100$} & ADRP 337 & ADRP 350 & ADRP 344 & ADRP 219 & ADRP 225 \\
\hline & & LEACH 520 & LEACH 506 & LEACH 28 & LEACH 285 \\
\hline & Static & Velocity $2 \mathrm{~m} / \mathrm{s}$ & Velo & Rayleigh $(4000)$ & Rayleigh(5000) \\
\hline & ADRP 19 & ADRP 194 & ADRP 192 & ADRP 112 & ADRP 112 \\
\hline$X=200 \quad Y=200$ & LEACH 217 & LEACH 187 & LEACH 165 & LEACH 111 & LEACH 95 \\
\hline
\end{tabular}



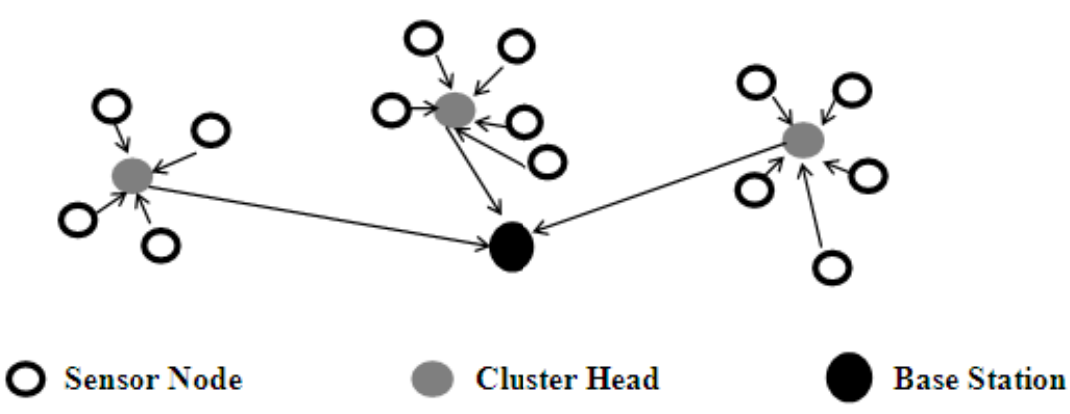

Figure 1. Cluster topology

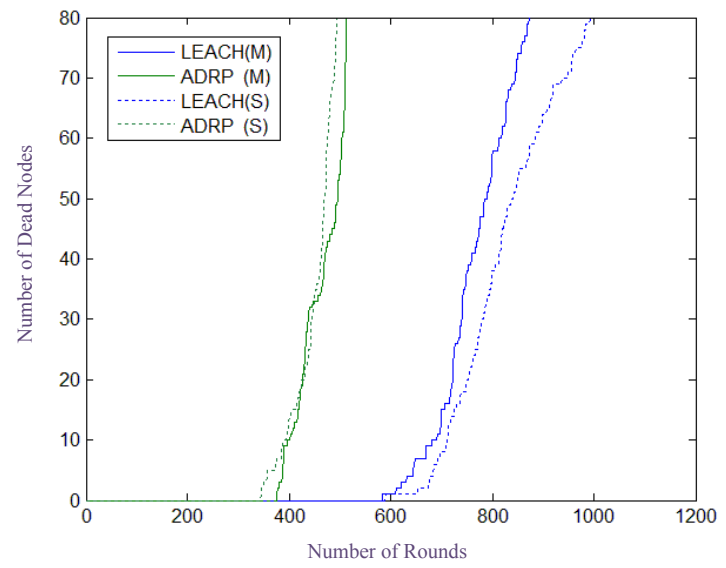

(a)

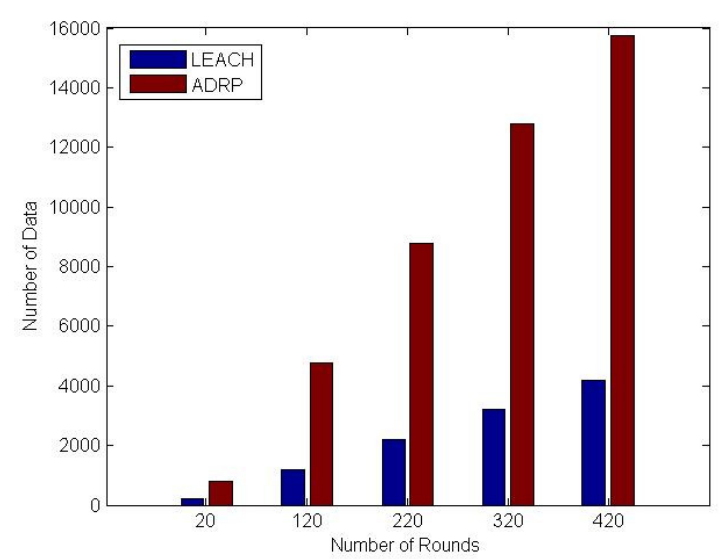

(c)

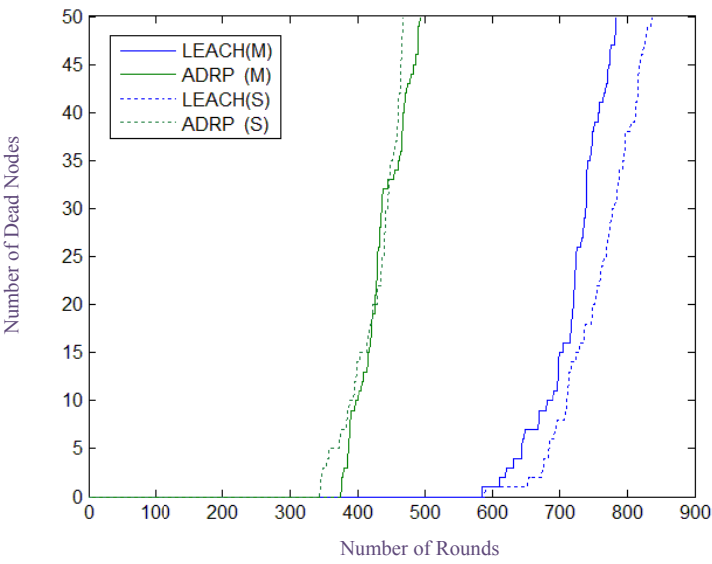

(b)

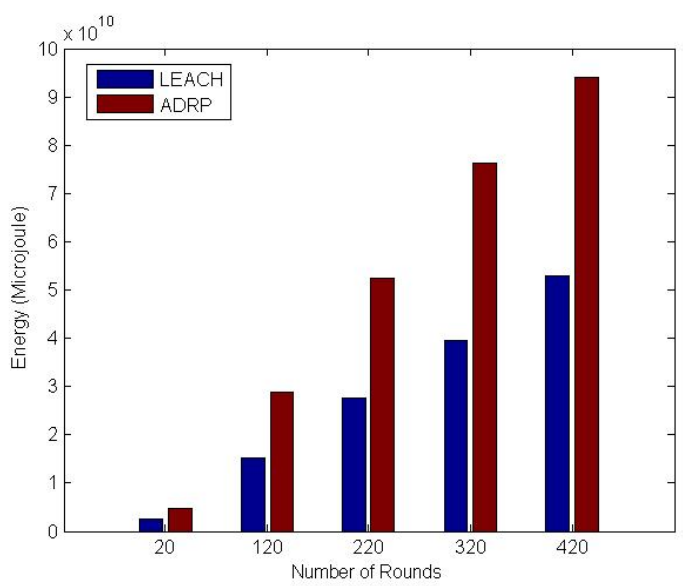

(d)

Figure 2. Mobile sensor nodes at speed 2m/s: (a) $80 \%$ of nodes are dead; (b) $50 \%$ of nodes are dead; (c) Number of received data message at base station; (d) Energy dissipation 


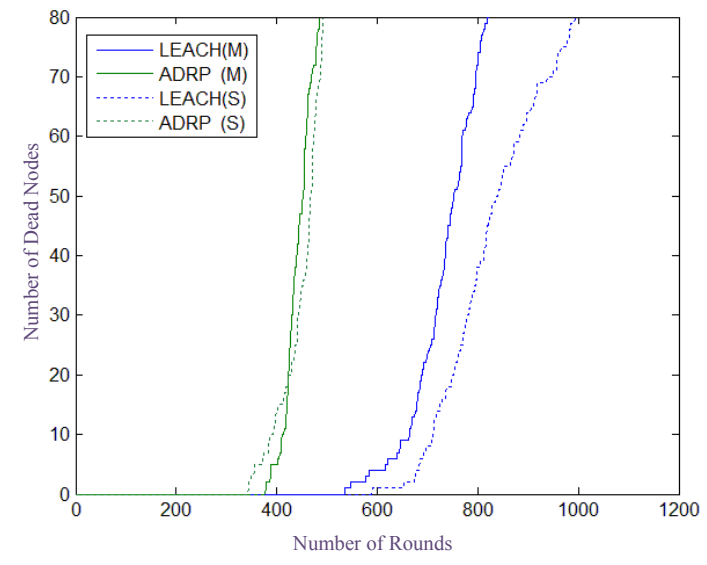

(a)

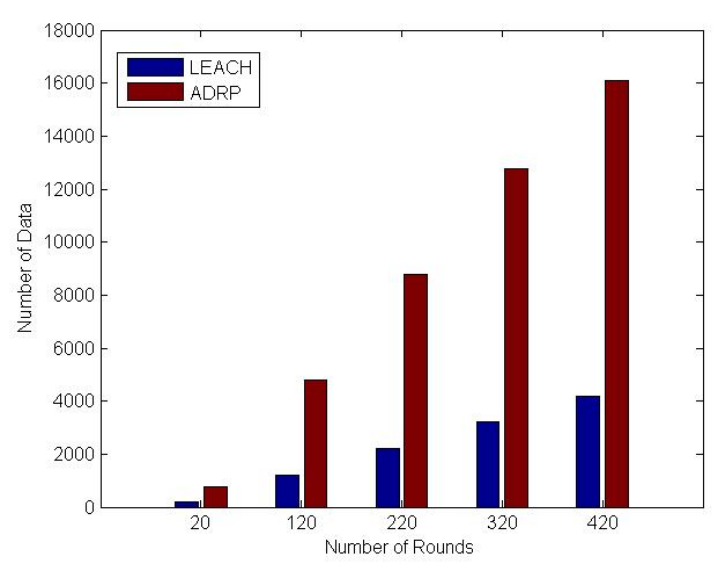

(c)

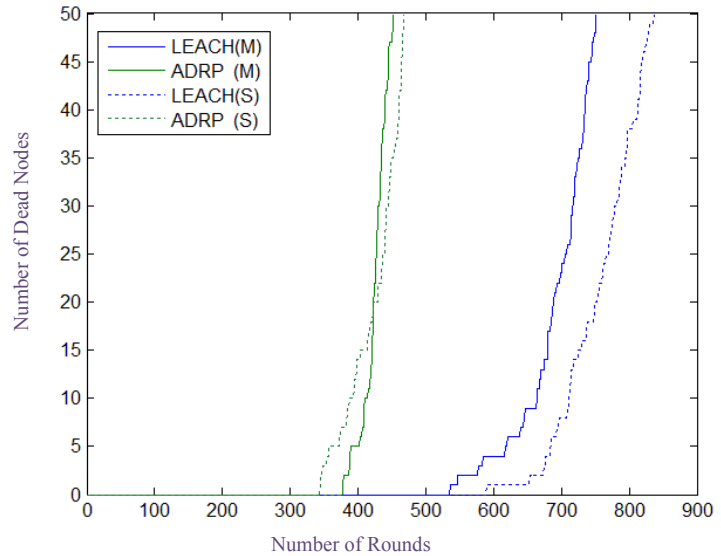

(b)

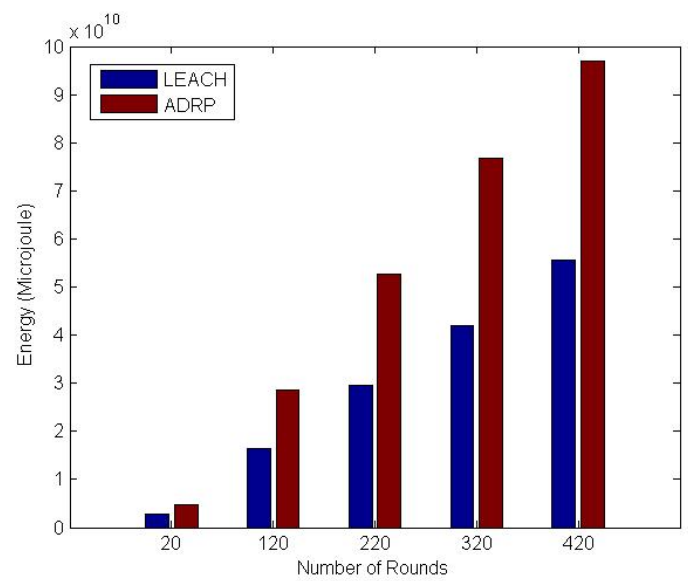

(d)

Figure 3. Mobile sensor nodes at speed $5 \mathrm{~m} / \mathrm{s}$ : (a) $80 \%$ of nodes are dead; (b) $50 \%$ of nodes are dead;

(c) Number of received data message at base station; (d) Energy dissipation 


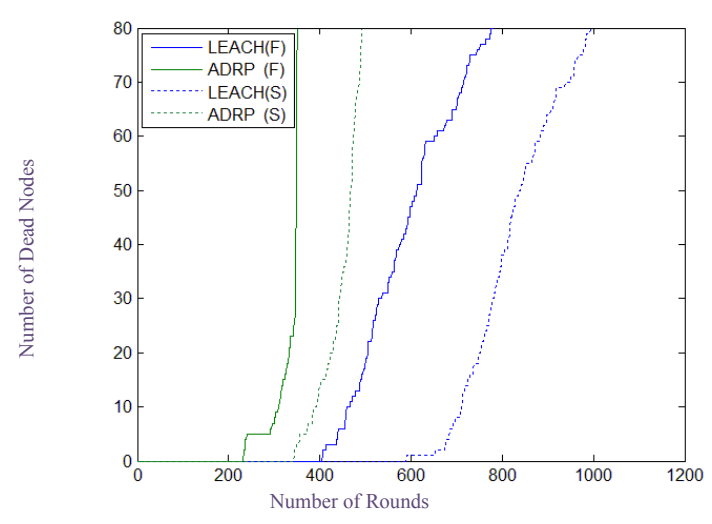

(a)

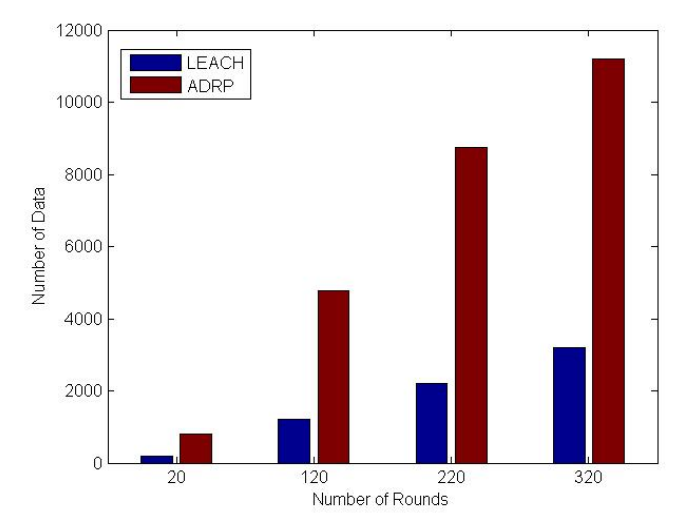

(c)

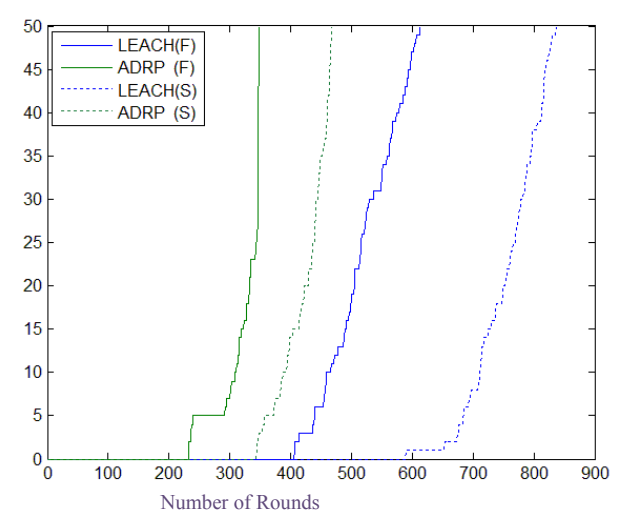

(b)

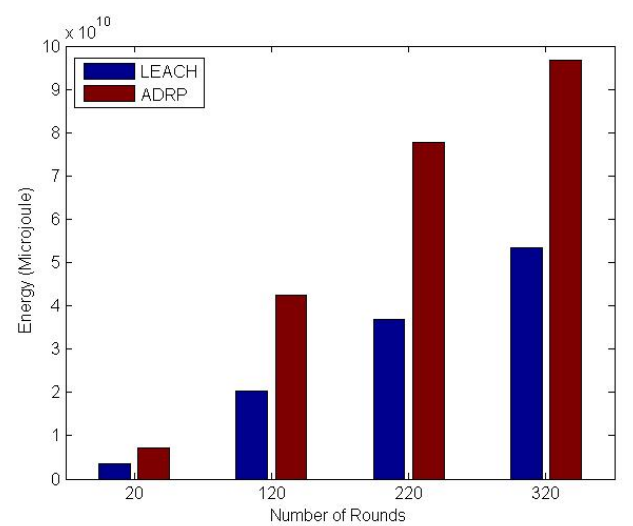

(d)

Figure 4. Rayleigh fading case 1: (a) $80 \%$ of nodes are dead; (b) $50 \%$ of nodes are dead;

(c) Number of received data message at base station; (d) Energy dissipation 


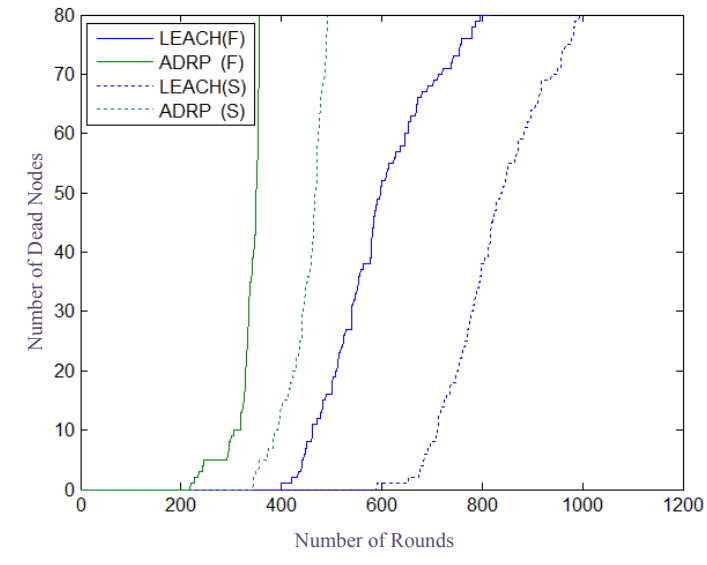

(a)

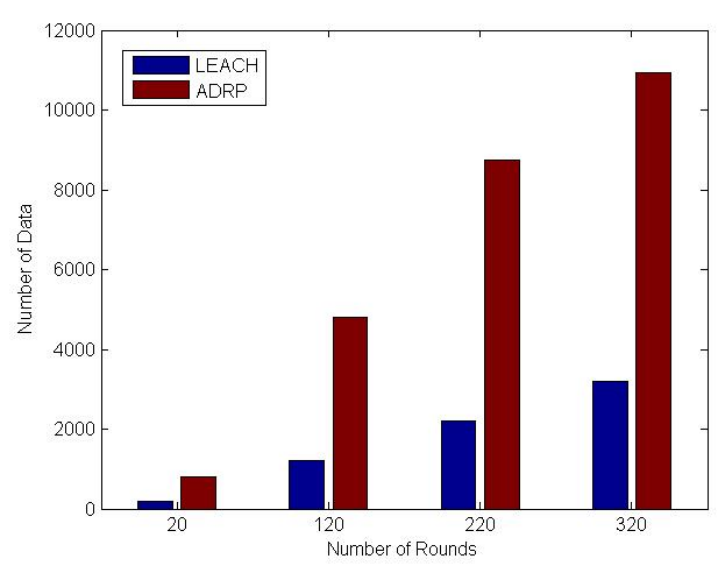

(c)

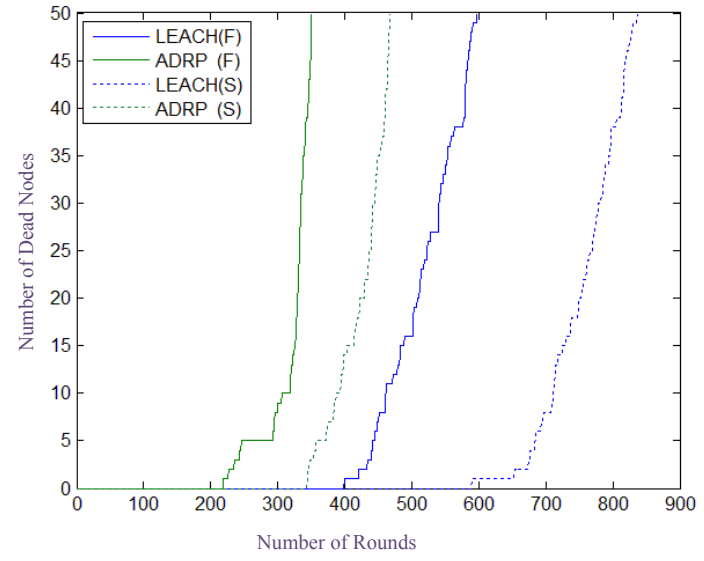

(b)

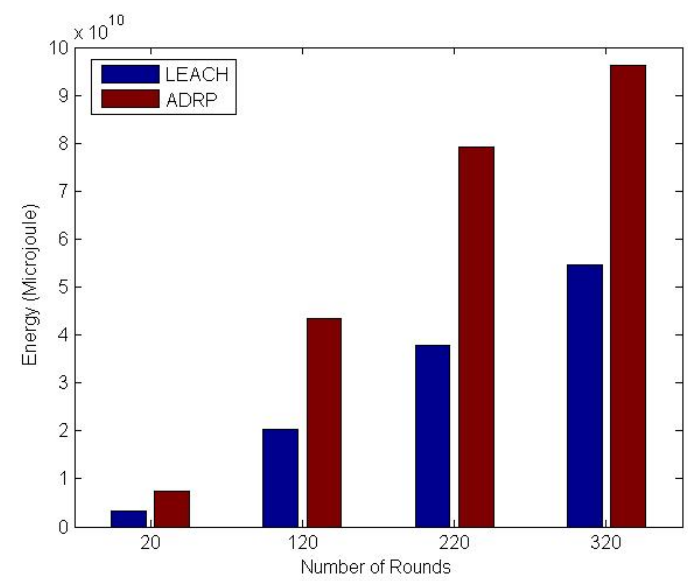

(d)

Figure 5. Rayleigh fading case 2: (a) $80 \%$ of nodes are dead; (b) $50 \%$ of nodes are dead;

(c) Number of received data message at base station; (d) Energy dissipation 